\title{
ESTIMATION OF TRIAL TO TRIAL VARIABILITY OF P300 SUBCOMPONENTS BY COUPLED RAO-BLACKWELLISED PARTICLE FILTERING
}

\author{
Delaram Jarchi, Bahador Makkiabadi, and Saeid Sanei
}

Centre of Digital Signal Processing, School of Engineering, Cardiff University, Wales, UK

\begin{abstract}
In this paper a new method based on Rao-blackwellised particle filtering for tracking variability of event relatedpotential (ERP) subcomponents in different trials is presented. The latency, amplitude, and width of each subcomponent is formulated in the state space model. Then, the observation is modeled as a linear function of amplitude and a nonlinear function of latency and width. The Raoblackwellised particle filtering is then applied for recursive estimation of the state of the system in different trials. To prevent generation of some invalid particles and also to have a reliable estimation in every situation, using some prior knowledge about some ERP subcomponents, a coupled Raoblackwellised particle filter is designed to detect variability of the desired ERP subcomponents. The method is applied to both simulated and real P300 data. The algorithm has the ability of tracking the variability of P300 subcomponents i.e. $\mathrm{P} 3 \mathrm{a}$ and $\mathrm{P} 3 \mathrm{~b}$, in single trials even in the low signal-to-noise ratio situations.
\end{abstract}

Index Terms - Event related potential (ERP), Raoblackwellised Particle filter, coupled RBPF, constrained RBPF, subcomponent, P300

\section{INTRODUCTION}

ERPs are voltage fluctuations in electroencephalogram (EEG) induced within the brain and are time locked to sensory, motor, or cognitive events [1][2]. They are used mainly by clinicians in order to assess a number of neurological disorders and cognitive processes. The ERP consists of a sequence of labeled positive and negative amplitude components. These components reflect various sensory, cognitive (e.g., stimulus evaluation) and motor processes that are classified on the basis of their scalp distribution and response to experimental stimulus. One common approach to analyze the ERP components is to average time-locked single-trial measurements. This approach assumes that the ERP wave is the same over time and the background EEG is a random process that will be attenuated by averaging over trials. This assumption may be reasonable for some but not all the cases. For example, changes in the degree of mental fatigue, habituation, or level

This work is supported by The Leverhulme Trust of attention of the subject can affect the ERPs. Therefore, averaging the EEG over a number of trials to obtain the ERP waveform implies loss of information related to trial-to-trial variability. An effective analysis of ERPs should then be based on single trial estimation. Several methods such as Wiener [3], maximum a posterior (MAP) [4] and Kalman filtering approaches $[5,6]$ have been used in single trial estimation. The main focus of most of these methods has been single trial estimation of ERP components, whereas here the aim is to separate and track the ERP subcomponents in different trials. In this paper we propose a method based on Rao-blackwellised particle filtering [7] to track the dynamic changes of amplitude, latency and shape of ERP subcomponents in different trials. The reminder of the paper is structured as follows. In section 2 the coupled Rao-blackwellised particle filter (CRBPF) is modeled and in section 3 the results of applying the CRBPF to both simulated and real data are provided. Finally section 4 concludes the paper.

\section{COUPLED RAO-BLACKWELLISED PARTICLE FILTERING}

In this section first the problem of tracking ERP subcomponents using Rao-blackwellised Particle Filtering (RBPF) from a single channel is formulated. Then, the required conditions and measures for coupling two RBPF is described. Since the aim is to track the latency, amplitude and width of ERP subcomponents, it is possible to form the state vector of the RBPF as:

$\mathbf{x}_{k}=\left[\begin{array}{lllllll}\mathbf{a}_{k}(1) & \mathbf{b}_{k}(1) & \mathbf{s}_{k}(1) & \ldots & \mathbf{a}_{k}(p) & \mathbf{b}_{k}(p) & \mathbf{s}_{k}(p)\end{array}\right]^{T}$

where $\mathbf{a}_{k}(i)$ are the amplitudes, $\mathbf{b}_{k}(i)$ are the latencies and $\mathbf{s}_{k}(i)$ is the width of the $i^{t h}, i=1, \ldots, p$ subcomponent in the $k^{t h}$ trial, and $p$ is the number of subcomponents. We can partition the state space variables into the linear and nonlinear state variables. It is possible to take the amplitudes out and form a matrix and find a linear relation between the observation and amplitudes and a nonlinear relation with respect to other variables. Therefore, regarding equation (1) the statespace and observation can be formulated as:

$$
\mathbf{x}_{k}^{1}=\left[\begin{array}{lllll}
\mathbf{b}_{k}(1) & \mathbf{s}_{k}(1) & \ldots & \mathbf{b}_{k}(p) & \mathbf{s}_{k}(p)
\end{array}\right]^{T}
$$




$$
\begin{gathered}
\mathbf{x}_{k}^{2}=\left[\begin{array}{lll}
\mathbf{a}_{k}(1) & \ldots & \mathbf{a}_{k}(p)
\end{array}\right]^{T} \\
\mathbf{x}_{k}=\mathbf{x}_{k-1}+\mathbf{v}_{k-1} \\
\mathbf{z}_{k}=\left[\begin{array}{lll}
f_{1}\left(\mathbf{x}_{k}^{1}\right) & \ldots & f_{p}\left(\mathbf{x}_{k}^{1}\right)
\end{array}\right]\left[\begin{array}{c}
\mathbf{a}_{k}(1) \\
\ldots \\
\mathbf{a}_{k}(p)
\end{array}\right]+n_{k}
\end{gathered}
$$

where

$$
f_{i}\left(\mathbf{x}_{k}^{1}, t\right)=f_{i}\left(\mathbf{b}_{k}(i), \mathbf{s}_{k}(i), t\right)=e^{-\frac{\left(t-\mathbf{b}_{k}(i)\right)^{2}}{2 \mathbf{s}_{k}^{2}(i)}}
$$

and $t$ denotes the time index and varies from the beginning of the ERP component to the end of ERP component. The whole time samples are used in the observation, therefore $f_{i}\left(\mathbf{x}_{k}^{1}, t\right)$ returns a time vector. Based on this model, each ERP component is modeled as sum of its subcomponents and each subcomponent is approximated by a Gaussian wave form. The formulated RBPF for tracking the dynamic changes of latency, amplitude and width of ERP subcomponents, may be applied to a single channel of ERP or EEG data. If we use some prior knowledge about the ERP components and initialize the particles close to the true posterior density, it is possible to track the ERP subcomponents using a single channel effectively. For example we can use the average ERP of a few first trials and then initialize particles. However, because there may exist some artifacts in the data, it is possible to lose the correct track of the ERP subcomponents. To have a reliable estimation, it is useful to have more RBPFs and try to make a connection between them using some prior knowledge or properties of ERP subcomponents. Therefore based on some properties of ERP subcomponents we can impose some constraints in order to prevent generation of invalid particles and to have a reliable estimation. Here, we design the CRBPF for separating and tracking P300 subcomponents. It is straightforward to generalize it for analysis of other ERP subcomponents. Each P300 subcomponent (P3a and $\mathrm{P} 3 \mathrm{~b}$ ) originates from a location inside the brain. $\mathrm{P} 3 \mathrm{a}$ is more frontal and P $3 b$ is in posterior parts. In every channel in the frontal part or the posterior part, the two components appear but in the frontal part P3a has a larger amplitude and in the posterior part the P $3 b$ has a larger amplitude. Since the aim is to separate P3a and P3b we can consider two channels each better highlights one of them. Therefore, Fz (among the electrodes in frontal part) and $\mathrm{Pz}$ (among the electrodes in posterior part) channels can be selected. From now on, the signal of channel Fz is considered as the first signal and RBPF1 is applied to the first signal and the signal of channel $\mathrm{Pz}$ is considered as the second signal and RBPF2 is applied to the second signal. It is possible to assume that the original shape of P3a and P3b in the brain is constant in every electrode and only the amplitude is different and we can assume a small delay in the latencies of P3a and P3b. In the initialization stage we randomly generate a relatively large number of particles with relatively large variances for the latency, amplitude and width. The initial particles for both RBPFs are exactly the same. In the subsequent iteration of RBPF the particles that are far from the true posterior density will be assigned a near zero weight and will be replaced by the particles with larger weights. At each iteration, when we want to draw particles from the prior density, we need to be careful about the required relation between the two RBPFs. As long as we have assumed that the shape of P3a or P3b in every channel remains the same, we can relate the pairs of particles with the same width for P3a and P3b. The CRBPF is designed based on these particle pairs. Because in the initialization part we produce the same particle pairs, it is possible to keep the width of a particle pairs for both P3a and P3b the same. In other words, at each iteration we just generate the same width for each pair of particles from the prior density for P3a and the same width for P3b. Using this relation between two RBPFs, the task of CRBPF is to estimate one width for every subcomponent in both channels, but with different amplitudes and approximately the same latency. After generating the widths and latencies from the prior density, we estimate the amplitudes using kalman filtering. Then by considering the properties of amplitude of $\mathrm{P} 3 \mathrm{a}$ and $\mathrm{P} 3 \mathrm{~b}$ on $\mathrm{Fz}$ and $\mathrm{Pz}$ channels, it is effective to remove the invalid particles. These invalid particles are the ones that the estimated amplitude of P3a on Pz channel is larger than the amplitude of P3a on Fz channel. Also the particles that the estimated amplitude of $\mathrm{P} 3 \mathrm{~b}$ on Fz channel is larger than the amplitude of $\mathrm{P} 3 \mathrm{~b}$ on $\mathrm{Pz}$ channel are considered invalid. Therefore, the weight of these invalid particles are set to zero in order not to have any contribution in any state of the system. Indeed, we may have another type of invalid particles at this stage. These invalid particles are those whose latency of P3b is shorter than the latency of P3a. Sometimes the P3a and P3b, have overlap over the scalp. So, the estimated subcomponents may be displaced in terms of latencies. It is effective to detect these invalid particles and set the weight of them to zero. The weight normalization can be done separately. Because always there is an uncertainty in the width value, the corresponding particles from the two RBPFs that have the same width value for P3a in both channels and also for P3b, should have high weights otherwise we need to decrease the weights of both particles. Therefore, it is possible to update the weight of both particle pairs according to:

$$
\begin{aligned}
& w 1_{k}^{i}=w 1_{k}^{i} \times \alpha \times \lambda^{\prime} \\
& w 2_{k}^{i}=w 2_{k}^{i} \times \alpha \times \lambda^{\prime}
\end{aligned}
$$

where

$$
\alpha=1-\left|w 1_{k}^{i}-w 2_{k}^{i}\right|
$$

and $\lambda^{\prime}$ is the penalty coefficient. In addition, resampling can be done simultaneously using a scaled weight of both particles pairs:

$$
w_{k}^{i}=\lambda \times w 1_{k}^{i}+(1-\lambda) \times w 2_{k}^{i}
$$




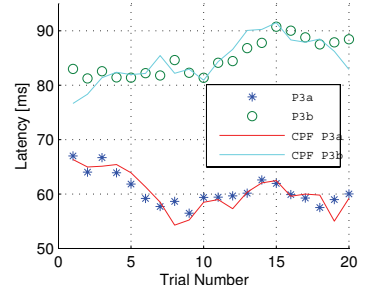

(a1)

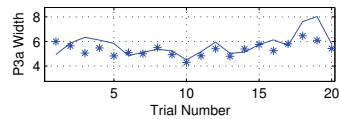

(c1)

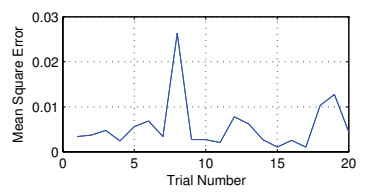

(e1)

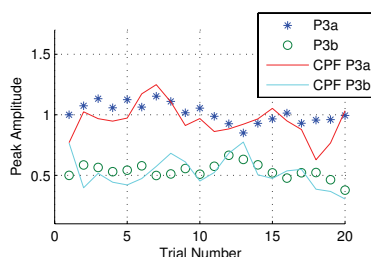

(b1)

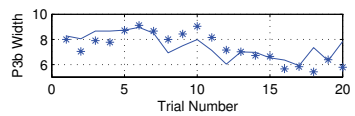

(d1)

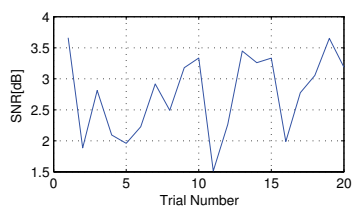

(f1)

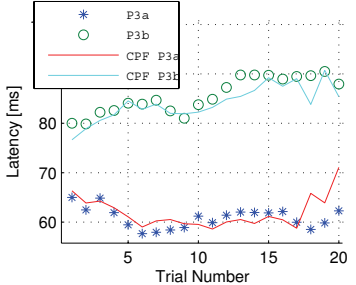

(a2)

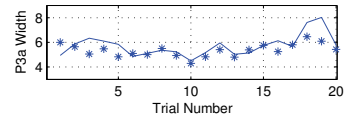

(c2)

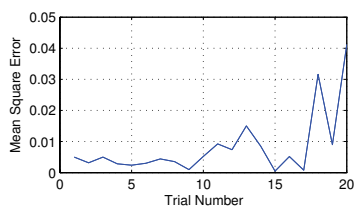

(e2)

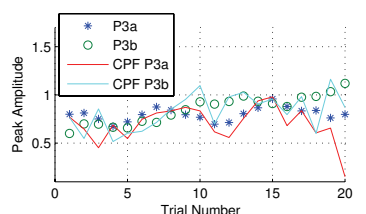

(b2)

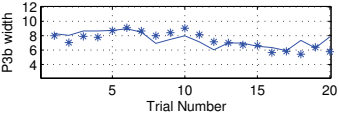

(d2)

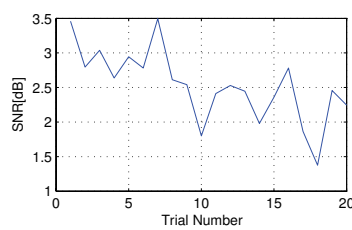

(f2)

Fig. 1. Tracking the latency and amplitude using CRBPF; The circle and asterisk show the actual values while the thick line is the result of tracking; (a1),(b1),(c1),(d1) tracking the latency, amplitude, width of P3a and width of P3b using the first signal respectively, (a2),(b2),(c2),(d2) tracking the latency, amplitude, width of P3a and width of P3b using the second signal respectively, (e1),(e2) The mean square error (MSE) obtained in different trials for the first and second signal, and (f1),(f2) The signal to noise ratio (SNR) calculated in different trials for the first and second signal.

where $\lambda$ controls the weight contribution of each particle pairs.

\section{EXPERIMENTAL RESULTS}

To show the effectiveness of the CRBPF, this method is applied to both simulated and real data. what follows is the description of the simulated and real data and the results of applying the CRBPF to both datasets.

\subsection{Simulated data}

Two Gaussian-shape waves as P3a and P3b are generated. The latencies, maximum amplitudes, and widths of the Gaussian waves are considered as the state variables. In each trial the state variables randomly change with uniform distribution. Two separate observations are generated separately from the separate state variables (except for the width variable that should be the same for P3a or P3b) but the required constraints for latency and amplitude explained in the paper for P300 subcomponents should be taken into account in order to have meaningful state variables. Therefore, it is tried to generate signals close to the real data in which the latency of P3a is smaller than the latency of P3b for both simulated signals and also the amplitude of $\mathrm{P} 3 \mathrm{a}$ in the first signal is bigger than the second signal and the amplitude of the P $3 \mathrm{~b}$ in the second signal is bigger than the first signal. The variance of the noise for the latency is 3 , and for the maximum amplitude is 0.1 , and for the width is 1 . Then, in each trial a random noise with a variance of 0.3 is added to the sum of generated Gaussians to form the observation. The results of tracking of latencies, maximum amplitudes, and widths of the generated Gaussians for the first and second RBPF are shown in Fig. 1. The mean square error (MSE) here is defined as:

$$
\mathbf{M S E}=\frac{1}{N} \sum_{i=1}^{N}\left(z_{\text {act }}(i)-z_{\text {est }}(i)\right)^{2}
$$

where $z_{a c t}$ is the original clean observation, $z_{\text {est }}$ is the estimated observation, and $N$ is the number of time samples.

\subsection{Real data}

The EEG data were recorded using a Nihon Kohden model EEG-F/G amplifier and Neuroscan Acquire 4.0 software. EEG activity was recorded following the international $10-$ 20 electrode setting system from 15 electrodes. The reference electrodes were linked to the earlobes. The impedances for all the electrodes are below $5 k \Omega$, the sampling frequency Fs $=$ $2 \mathrm{kHz}$, and the data were subsequently bandpass filtered $(0.1$ $-70 \mathrm{~Hz}$ ). This frequency range was chosen to be compatible with [8]. Subjects were required to sit alert and still with their eyes closed to avoid any interference. Also, to avoid any 


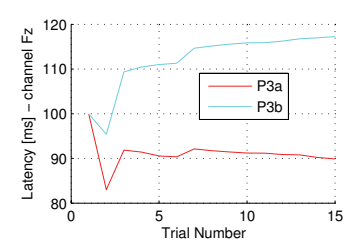

(a)

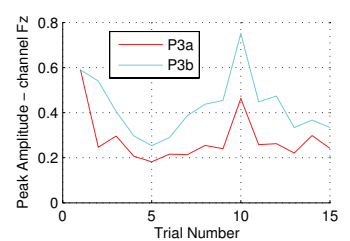

(b)

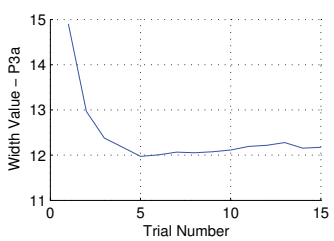

(e)

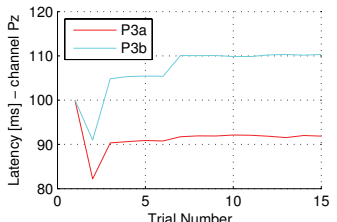

(c)

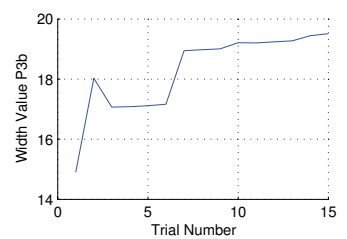

(f)

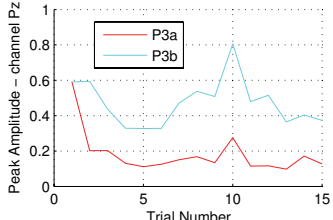

(d)

Fig. 2. Tracking the latency and amplitude using CRBPF; (a),(b) tracking the latency and amplitude of simulated P3a and P3b from Channel Fz using CRBPF, (c),(d) tracking the latency and amplitude of the simulated P3a and P3b from Channel Pz using CRBPF, (e) tracking width of P3a, (f) tracking width of P3b.

muscle artifact, the neck was firmly supported by the back of the chair. The feet were rested on a footstep. The stimuli were presented through ear plugs inserted in the ear. Forty rare tones $(1 \mathrm{kHz})$ were randomly distributed amongst 160 frequent tones $(2 \mathrm{kHz})$. Their intensity was $65 \mathrm{~dB}$ with 10 and 50-milliseconds duration for rare and frequent tones, respectively. The subjects were asked to press a button as soon as they heard a low tone $(1 \mathrm{kHz})$. The ability to distinguish between low and high tones was confirmed before the start of the experiment. The task is designed to assess basic memory processes. ERP components measured in this task included N100, P200, N200, and P3a and P3b. The channel Fz and Pz are selected as the first and second signal respectively. The average ERP of a few first trials is used to initialize particles of both RBPFs. Then the CRBPF is applied for tracking the latency, amplitude, and width of the P3a and P3b on both channels. The results of applying the CRBPF to track P3a and P3b variability in the 15 trials are shown in Fig. 2.

\section{DISCUSSION AND CONCLUSIONS}

In this paper a new method is proposed to separate and track the ERP subcomponents in different single trials. The coupled Rao-blackwellised particle filter proposed in this paper uses the concept of Rao-blackwellised particle filter, combines two RBPFs and imposes a constraint on the state variables to have a reliable estimation and to use the required number of particles effectively. The particle initialization with the help of average ERP was useful to move the particles toward the right part of the posterior density. Therefore, the algorithm is capable of estimating ERP components and subcomponents in single trials. Here for the first time all variations of the P300 subcomponents (P3a and P3b), including latency, amplitude, and width, have been considered from single trials. This sin- gle trial estimation method is helpful in some applications such as mental fatigue in which tracking dynamical changes of different trials can determine the stage of fatigue.

\section{REFERENCES}

[1] S. Sanei and J. Chambers, EEG signal Processing, John Wiley and Sons, 2007.

[2] D. Friedman, Y. M. Cycowicz, and H. Gaeta, "The novelty p3: an event-related brain potential (ERP) sign of the brain's evaluation of novelty," Neuroscience and Biobehavioral Reviews, vol. 25, pp. 355-373, 2001.

[3] S. Cerutti, V. Bersani, A. Carrara, and D. Liberati, "Analysis of visual evoked potentials through wiener filtering applied to a small number of sweeps," J. Biomed. Eng., vol. 9, pp. 3-12, 1983.

[4] W. A. Truccolo, K. H. Knuth, A. Shah, S. L. Bressler, C. E. Schroeder, and M. Ding, "Estimation of single-trial multicomponent ERPs: Differentially variable component analysis (dvca)," Biological Cybernetics, vol. 89, pp. 426-438, 2003.

[5] M. V. Spreckelsen and B. Bromm, "Estimation of single-evoked cerebral potentials by means of parametric modeling and kalman filtering," IEEE Trans. Biomed. Eng, vol. 35, no. 9, pp. 691-700, 1988.

[6] S. D. Georgiadis, P. O. Ranta-aho, M. P. Tarvainen, and P. A. Karjalainen, "Single-trial dynamical estimation of event-related potentials: a kalman filter-based approach," IEEE Trans. Biomed. Eng, vol. 52, no. 8, pp. 1397-1406, 2005.

[7] M. S. Arulampalam, S. Maskell, N. Gordon, and T. Clapp, "A tutorial on particle filters for online nonlinear/non-gaussian bayesian tracking," IEEE Trans. Signal Processing, vol. 50, no. 2, 2002.

[8] B. Karoumi, A. Laurent, and F. Rosenfeld, "Alteration of event related potentials in siblings discordant for schizophrenia," Schizophrenia Research, vol. 41, pp. 325-334, 2000. 